\title{
Effects of the neo-X chromosome on genomic signatures of hybridization in Rumex hastatulus
}

\author{
Felix Beaudry ${ }^{1}$, Joanna Rifkin ${ }^{1}$, Amanda Peake ${ }^{1}$, Deanna Kim ${ }^{1}$, Madeline Jarvis-Cross ${ }^{1}$, \\ Spencer Barrett ${ }^{1}$, and Stephen Wright ${ }^{1}$
}

${ }^{1}$ University of Toronto

February 15, 2022

\begin{abstract}
Natural hybrid zones provide opportunities for studies of the evolution of reproductive isolation in wild populations. Although recent investigations have found that the formation of neo-sex chromosomes is associated with reproductive isolation, the mechanisms remain unclear in most cases. Here, we assess the contemporary structure of gene flow in the contact zone between largely allopatric cytotypes of the dioecious plant Rumex hastatulus, a species with evidence of sex chromosome turn-over. Males to the west of the Mississippi river, USA, have an X and a single Y chromosome, whereas populations to the east of the river have undergone a chromosomal rearrangement giving rise to a larger $\mathrm{X}$ and two $\mathrm{Y}$ chromosomes. Using reducedrepresentation sequencing, we provide evidence that hybrids form readily and survive multiple backcross generations in the field, demonstrating the potential for ongoing gene flow between the cytotypes. Cline analysis of each chromosome separately captured no signals of difference in cline shape between chromosomes. However, principal component regression revealed a significant increase in the contribution of individual SNPs to inter-cytotype differentiation on the neo-X chromosome, but no correlation with recombination rate. Cline analysis revealed that the only SNPs with significantly shallower clines than the genome-average were located on the neo-X. Our data are consistent with a role for neo-sex chromosomes in reproductive isolation between R. hastatulus cytotypes. Our investigation highlights the importance of studying plant hybrid zones in species with sex chromosomes for understanding mechanisms of reproductive isolation and the role of gene flow in the spread of neo-X chromosomes.
\end{abstract}

\section{Hosted file}

Beaudry et al. RumexHybridZone.220207.pdf available at https://authorea.com/users/460658/ articles/556563-effects-of-the-neo-x-chromosome-on-genomic-signatures-of-hybridizationin-rumex-hastatulus 